\title{
A Comparative Study of Support Vector Machine and Artificial Neural Network for Option Price Prediction
}

\author{
Biplab Madhu, Md. Azizur Rahman*, Arnab Mukherjee, Md. Zahidul Islam, Raju Roy, \\ Lasker Ershad Ali \\ Mathematics Discipline, Science, Engineering and Technology School, Khulna University, Khulna, Bangladesh \\ Email: ^mdazizur@math.ku.ac.bd
}

How to cite this paper: Madhu, B., Rahman, Md.A., Mukherjee, A., Islam, Md.Z., Roy, R. and Ali, L.E. (2021) A Comparative Study of Support Vector Machine and Artificial Neural Network for Option Price Prediction. Journal of Computer and Communications, 9, 78-91.

https://doi.org/10.4236/jcc.2021.95006

Received: May 1, 2021

Accepted: May 25, 2021

Published: May 28, 2021

Copyright $\odot 2021$ by author(s) and Scientific Research Publishing Inc. This work is licensed under the Creative Commons Attribution International License (CC BY 4.0).

http://creativecommons.org/licenses/by/4.0/ (c) (i) Open Access

\begin{abstract}
Option pricing has become one of the quite important parts of the financial market. As the market is always dynamic, it is really difficult to predict the option price accurately. For this reason, various machine learning techniques have been designed and developed to deal with the problem of predicting the future trend of option price. In this paper, we compare the effectiveness of Support Vector Machine (SVM) and Artificial Neural Network (ANN) models for the prediction of option price. Both models are tested with a benchmark publicly available dataset namely SPY option price-2015 in both testing and training phases. The converted data through Principal Component Analysis (PCA) is used in both models to achieve better prediction accuracy. On the other hand, the entire dataset is partitioned into two groups of training (70\%) and test sets (30\%) to avoid overfitting problem. The outcomes of the SVM model are compared with those of the ANN model based on the root mean square errors (RMSE). It is demonstrated by the experimental results that the ANN model performs better than the SVM model, and the predicted option prices are in good agreement with the corresponding actual option prices.
\end{abstract}

\section{Keywords}

Machine Learning, Support Vector Machine, Artificial Neural Network, Prediction, Option Price

\section{Introduction}

The financial market may be regarded as the propellant of any country's economy. However, the relationship between the currency market and the country's ${ }^{\star}$ Corresponding author. 
economy is really complicated. Identifying this relationship is one of the most important parts of any money investment decision making framework [1] [2] [3]. In this context, derivatives such as option became very significant part of the financial market over the past few decades. Option is a financial contract between two parties that provides the buyer (the owner or holder) of the option the right (but not an obligation) to buy or sell the underlying asset for the settled price (strike price) on or before expiring time (maturity time) of the contract, depending on the form of the option. There exist two fundamental types of options namely, call option (option for buying) and put option (option for selling). The seller and buyer can protect their financial risk with the help of option contract. For this reason, the problem of option price prediction has received considerable attention from scientific community. It is important to predict option price to know rigorously the future trends of financial market. However, accurately forecasting option price is a major challenge in stock market as it follows a complex pattern and shows stochastic behavior. In addition, it has been pointed out that forecasting option value is dynamic, sophisticated and chaotic in nature [4]. Thus, the study on the option price prediction is very worthy.

Several researchers have worked out to predict option value by adopting some ancient and innovative techniques. Examples of such techniques include movingaverage (MA), regression (R), auto-regression (AR), AR moving-average (ARMA), and AR integrated moving-average (ARIMA). In these techniques, the correlated data is used in the process and different types of assumption are required for different parametric specifications, and consequently, the standard of the prediction results degrades [5]. In addition, these models are not capable of handling non-stationary time series data. Thus, it is essential to develop updated models with higher capacities for accomplishing the task of forecasting. Soft computing techniques can be used in this regard that covers mimic biological processes. These techniques include Artificial Neural Network (ANN), Numerical Rationale (NR), Support Vector Machine (SVM), Molecule Swarm Improvement (MSI), etc. Among these models, ANN and SVM models have been widely used in a variety of fields of science and technology, including prediction problems.

The objective of this paper is to carefully examine, compare and analyze the performance of two highly promising and frequently used soft computing techniques of SVM and ANN for predicting option price. For this reason, both techniques are first evaluated individually and the predicted results are compared with the actual results. Then, the results of both techniques are compared with each other. The experimental outcomes indicate that the ANN model shows better performance than the SVM model for the prediction of option price. The rest part of this paper is organized as follows. The next section (Section 2) reviews some related work. A brief introduction of NN, ANN and SVM models is presented in Section 3. Section 4 contains the details of the dataset and the methodology to accomplish the task of this paper. The experimental results and the discussion of the results with comparison are reported in Section 5. And the final section (Section 6) offers the conclusion of the paper. 


\section{Related Work}

Various researchers have been designed and developed different architectures with the help of modern technology for effectively handling prediction problems. Some of the related literatures are reviewed in this section. The ANN model was successfully applied for the forecasting of different option prices by Liu in 1996 [6], Yao et al. in 2000 [7] and Andreou et al. in 2008 [8]. Besides that, the ANN based predicting frameworks for stock prices forecasting were reported in [9] [10] [11]. Moreover, some authors paid their attention to developing forecasting models using a variety of strategies. Saxena proposed a hybrid model for estimating the value of option by integrating the ANN model and Black Scholes model [12]. He tested his model with European-style CNX Nifty Options traded at National Stock Exchange of India Ltd. and showed that the hybrid model outperforms the Black Scholes model. While Mitra combined the ANN model and Black Scholes model with the S\&P CNX Nifty index call options in India and reported its superior performance than the original Black Scholes model [13]. In addition, Lajbcygier and Connor proposed a hybrid algorithm by using the ANN model and bootstrap algorithm to improve the option pricing [14]. On the other hand, Hassan et al. studied on forecasting the financial market behavior through a fusion model by combining Hidden Markov Model, ANN and Genetic Algorithm [15]. Their hybrid approach performed well compared to the conventional forecast method.

Kara et al. developed ANN and SVM models and compared their effectiveness for the prediction of the direction of movement in the daily Istanbul financial stock exchange (ISE) National 100 record [16]. They showed that the ANN model performs better than the SVM model in this specific case. In [17], Zhu et al. used a component based ANN to predict one-step ahead stock index increments. They trained ANN with the data of stock returns and volumes from NASDAQ, DJIA and STI list. Their experimental results demonstrated that expanded ANN models with corporate greed volumes can improve the performance of stock index increments prediction under medium and long-term horizons. Chen et al. studied on forecasting the six significant Asian stock markets by adopting SVM and back propagation ANN [18]. They showed a comparison between SVM and back propagation ANN and found the better performance of both models compared to other existing models. Li et al. established a dynamic prediction model for surface movements through the knowledge of the time series analysis and the theory of SVM [19].

Hutchinson et al. proposed a nonparametric procedure for pricing and hedging derivative asset through learning networks and compared its superiority with the Black Scholes option pricing model [20]. Kim applied SVM to predicting the stock price index and compared its capability with the back propagation ANN [21]. His experiments showed that SVM can be considered as a promising alternative technique for stock market prediction. Yoshihara et al. used a recurrent deep NN framework with real-world data of Nikkei companies for the predic- 
tion of stock market trend [22]. Its performance was compared with SVM and Deep Belief Network (DBN) and found a better forecast than both SVM and DBN. Lekhani proved by experiments that ANN is better and more accurate model than the Support Vector Regression (SVR) model for the stock prediction [23]. Recently, Madhu et al. adopted various kernels in the SVR to predict option price [24]. Their experiments illustrated that the SVR with Gaussian Kernel performs well compared to other kernel functions.

\section{Methods of Study}

In this section, we give a brief introduction of the methods under consideration in this study. The Neural Network (NN), ANN with biological NN and SVM models are briefly discussed in the following subsections consecutively.

\subsection{Neural Network}

The term Neural Network (NN) can be specified as a logical model, which is designed based on the human brain. The human brain contains interconnected nerve cells named neurons. In fact, the human brain holds about 10 billion neurons and 60 trillion connections, synapses, between them. A nerve cell or neuron consists of three modules-the summing function, the activation function, and the output. The term "Neural" comes from the "neuron" or nerve cells, the basic functional unit of the human (animal) nervous system that exist in the brain and other parts of the human (animal) body. There are mainly three parts in a typical nerve cell or neuron of a human brain such as dendrite, cell body and axon. There is also another important part called Synapses. The definition of each part of a neuron is given below:

Dendrite: It accepts signals from other neurons.

Cell body (Soma): It sums all the arriving signals to produce input.

Axon: When the sum influences a threshold value, neuron fires and the signal journeys down the axon to the other neurons.

Synapses: It is the point of interconnection of one neuron with other neurons. The amount of signal transmitted depends upon the strong point (synaptic weights) of the connections. The connections can be preclusive (decreasing strength) or manifest (increasing strength) in nature.

In general, $\mathrm{NN}$ is a highly interconnected network of billions of neurons with trillion of interconnections between them which influence to run the human body. A typical neuron with its different parts is shown in Figure 1.

\subsection{Artificial Neural Network with Biological Neural Network}

The dendrites of the biological NN are analogous to the weight inputs based on their synaptic interconnection in the ANN. The cell body is analogous to the artificial neuron unit in the ANN which comprises with the summation and threshold unit. On the other hand, the axon carries the output which is also analogous to the output unit in the case of ANN. Therefore, the ANN model is worked 


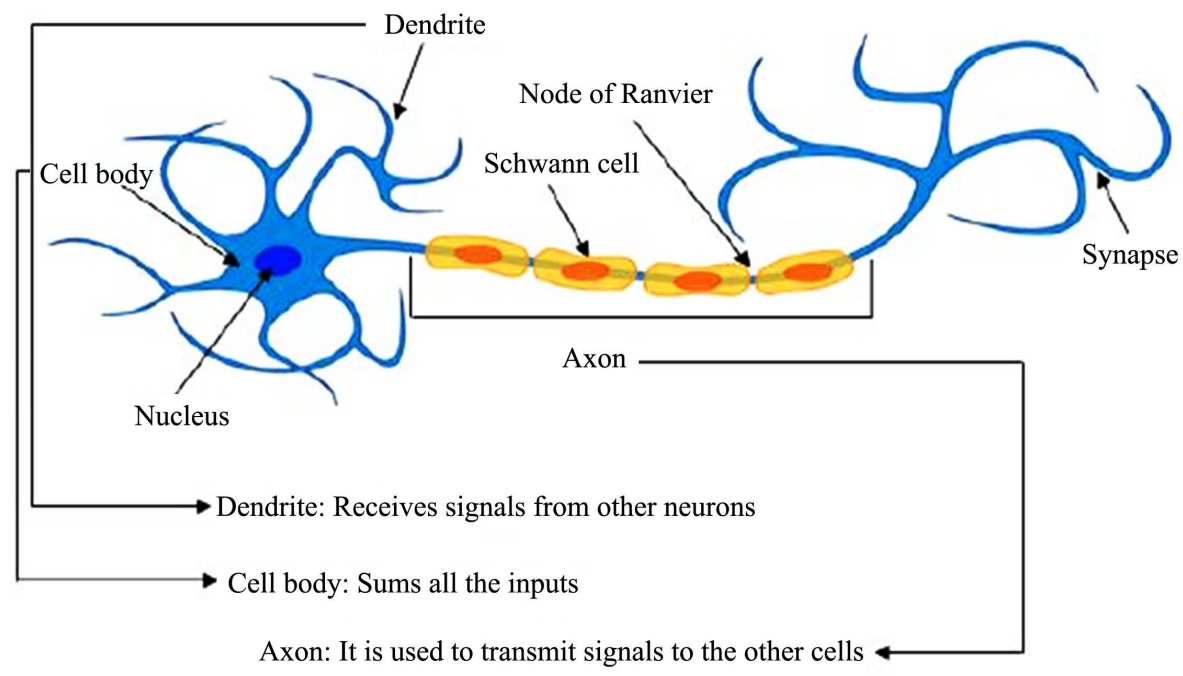

Figure 1. A typical neuron with its different parts [25].

based on the basic working functions of the biological neurons. A biological ANN is presented graphically in Figure 2.

The ANN is a biologically enthused network of artificial neurons, which is executed on a computer basis to perform certain tasks such as clustering, classification, pattern detection, etc. In fact, the architecture of ANN is designed based on the approach and act of the human brain's neurons. The ANN contains nonlinear and non-parametric units which process information, knowledge, intelligence, instruction etc. It is a computational method intended by the study of the brain and nervous system. The ANN follows the structure and operations of the three-dimensional lattice of network among brain cells. The network learns gradually by smoothing the connections between electronic neurons in its system. The learning process of the network can be deliberated like as a child learns to identify patterns, shapes and sounds, and discerns among them. For example, the child has to be illuminated to a number of examples of a particular type of animals for her to be skilled to recognize that type of animal later on. In addition, the child has to be irradiated to different types of animals for her to be capable to differentiate among animals. There are many different kinds of ANN architectures and several algorithms for network training. The choice of the ANN model depends on the prior knowledge of the system to be modeled. A feed forward neural network with one hidden layer is adopted in this study to forecast the option price in the stock market.

\subsection{Support Vector Machine}

The SVM was first applied by Vladimir N. Vapnik and A. Y. Chervonenkis in the year of 1963 [24]. It is a classifier of supervised learning, also known as a support vector network. The SVM was originally designed for classification, regression and outlier detection; however, later it has expanded in other directions. Indeed, it is a classifier derived from the theory of statistical learning based on 


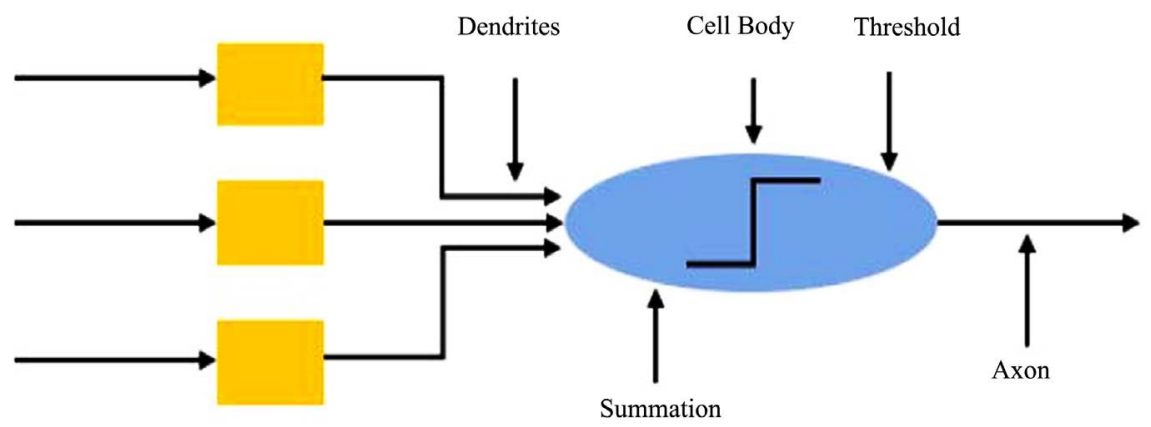

Figure 2. Biological Artificial Neural Network (ANN).

structural risk minimization that is used to maximize the accuracy of predictions and to reduce the problem of overfitting. It can efficiently perform classification of both linear and nonlinear problems and work well for many practical problems. The basic idea of SVM is to generate a line or a hyperplane that separates the data into classes.

In the linear SVM model, each input data is plotted as a point in the n-dimensional space where $\mathrm{n}$ is input dimensions. After that, the classification task is accomplished by getting the hyperplane which differentiate the data into two classes. Figure 3 represents the SVM margin and hyperplane with trained samples classes. Let us consider a linear classifier (or, hyperplane) [24]:

$$
f(x)=w^{\mathrm{T}} x+b
$$

In the above equation, $x$ represents the input feature vector of the classifier, $W$ indicates the weight vector, $w^{T}$ is the transpose of the weight vector, and $b$ holds for the hyperplane position. The linear Equation (1) represents a straight line, a plane and a hyperplane, if the input vector is 2-dimensional, 3-dimensional, and more than 3-dimensional, respectively. The SVM model finds an optimal hyperplane for the classification of two classes. Let the equation of hyperplane is $w^{\mathrm{T}} x+b=0$. The distance between $w \cdot x+b=+1$ and $w \cdot x+b=-1$ is the margin of this hyperplane. By using the formula to calculate the distance between two straight lines, we get the following margin:

$$
m=\frac{2}{\|w\|}
$$

On the other hand, the SVM model performs a classification task for nonlinear problems by adopting the kernel function. In this case, the original input vector projects into the higher dimensional feature space in a nonlinear manner. After transforming data into the new higher space, the new space is searched for a linear separating hyper-plane. To get a nonlinear SVM regression model, the dot product $x_{1}^{\mathrm{T}} \cdot x_{2}$ is exchanged with a nonlinear kernel function $K\left(x_{1}, x_{2}\right)=\left\langle\varphi\left(x_{1}\right), \varphi\left(x_{2}\right)\right\rangle$ where, $\varphi(x)$ is a transformation operator that maps $x$ to a high-dimensional space. There are many kernel functions in the literature and some popular kernel functions are given below:

1) Linear kernel function is expressed by $K\left(x_{j}, x_{k}\right)=x_{j}^{\mathrm{T}} x_{k}$ 


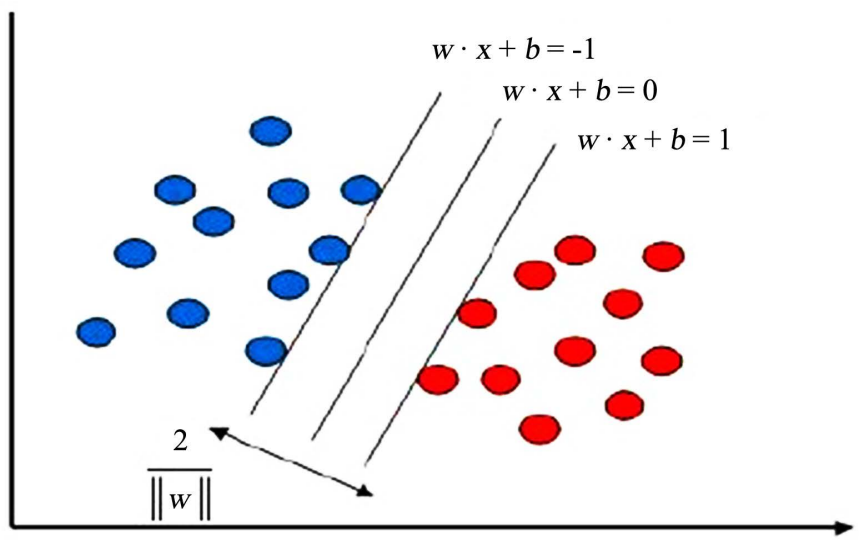

Figure 3. Support vector machine margin and hyperplane with trained samples classes [24].

2) Gaussian kernel function is presented by $K\left(x_{j}, x_{k}\right)=\exp \left(-\left\|x_{j}-x_{k}\right\|^{2}\right)$

3) Polynomial kernel function is figured out by $K\left(x_{j}, x_{k}\right)=\left(1+x_{j}^{\mathrm{T}} x_{k}\right)^{q}$

Here $x_{i}$ and $x_{j}$ represent the support vectors. Actually, support vectors are the input vectors of SVM classifier that just touch the boundary of the margin of the hyperplane. Simply, support vectors are the information points that are closest to the decision surface.

\section{Data and Methodology}

To conduct experiments, we collect data from the Yahoo Finance community named SPY option price-2015 [24] [26]. Actually, the Yahoo Finance community is a publicly available excellent source of financial data, which has received considerable attention from the research community to deal with very difficult and challenging problems. Both models are tested with the same dataset. The sample of data is presented in Table 1. From the table, it is observed that there are 4742 data with seven input variables in the dataset. It is difficult to conduct the experiments with this high dimension data. For this reason, we convert original data partially through Principal Component Analysis (PCA). The detailed process of adopting PCA for dimension reduction is presented in our earlier work [24]. The converted dataset contains 4742 data with two variables and based on it, the experimental task is accomplished.

The performance measure indicator of our study is root mean square errors (RMSE). Indeed, the RMSE for both models under consideration is calculated based on Equation (4). The predicting price error of SVM and ANN for each option can be expressed as follows:

$$
E_{i}=M_{i}-P_{i}
$$

In the above equation, parameter $E_{i}$ illustrates the error of the prediction value of an option for input $i, M_{i}$ denotes the market value of that option, and $P_{i}$ represents the predicted option price. We apply the formula (Equation (3)) for calculating the predicting error for each option. Since the errors can be either 
Table 1. Market value data of Yahoo option [24] [26].

\begin{tabular}{ccccccccc}
\hline S/N & $\begin{array}{c}\text { Strike } \\
\text { Price }\end{array}$ & $\begin{array}{c}\text { Stock } \\
\text { Price }\end{array}$ & High/Low & $\begin{array}{c}\text { Price } \\
(\text { Call/Put) }\end{array}$ & Vega $(\Lambda)$ & $\begin{array}{c}\text { Gamma } \\
(\Gamma)\end{array}$ & $\begin{array}{c}\text { Rho } \\
(\rho)\end{array}$ & Volatility \\
\hline 01 & 81.732 & 120 & 82 & 87.161 & $5.10361 \mathrm{e}-04$ & 0.0129431 & 0.992285 & 2.19259 \\
02 & 76.731 & 125 & 77 & 82.162 & $5.84782 \mathrm{e}-04$ & 0.0134769 & 0.991642 & 2.03664 \\
03 & 71.734 & 130 & 72 & 77.173 & $6.73353 \mathrm{e}-04$ & 0.014018 & 0.988927 & 1.88642 \\
04 & 66.735 & 135 & 67 & 72.171 & $7.79894 \mathrm{e}-04$ & 0.0145372 & 0.987126 & 1.74082 \\
05 & 61.736 & 140 & 62 & 67.172 & $9.09641 \mathrm{e}-04$ & 0.0150652 & 0.989221 & 1.60017 \\
06 & 56.737 & 145 & 57 & 62.183 & 0.00103995 & 0.0155902 & 0.989185 & 1.46322 \\
07 & 51.731 & 150 & 52 & 57.184 & 0.00167137 & 0.0161117 & 0.984988 & 1.33017 \\
08 & 46.732 & 155 & 47 & 52.192 & 0.00172959 & 0.0166296 & 0.983581 & 1.20041 \\
09 & 41.733 & 160 & 42 & 47.193 & 0.00186875 & 0.0171404 & 0.982901 & 1.07365 \\
. &. &. &. &. &. &. &. &. \\
. &. &. &. &. &. &. &. &. \\
&. &. &. &. &. &. &. &. \\
4742 & 0 & 265 & 0 & 0 & 0 & 0 & 0 & 0.11372 \\
\hline
\end{tabular}

positive or negative, we use the square amount of these predicting errors. Therefore, the formula for enumerating RMSE for whole data is presented in Equation (4).

$$
\mathrm{RMSE}=\sqrt{\frac{\sum_{i=1}^{n} E_{i}^{2}}{n}}
$$

In the Equation (4), variable $n$ is the number of samples. The smaller value of RMSE means the smaller predicting errors, and consequently, it means better option price prediction.

The purpose of this study is to explore the best learning method between ANN and SVM for option price prediction. For both models, the input dataset is divided into two parts - the training dataset and the testing dataset. In fact, the training dataset contains $70 \%$ of the data and the remaining $30 \%$ of the data are considered for testing. The dataset is transformed to get relevant attributes according to the input format of ANN and SVM. We use experiments through the trial and error method to get the minimum RMSE for each model. To get the output, we use some input variables which are listed in Table 2. There are two main things to keep in mind to designing the architecture of this study. Firstly, we need to know the architecture of the SVM model. Different kinds of kernel functions are used in the process of SVM model to minimize the prediction errors. Secondly, we regulate the architecture of ANN model by adopting a feed forward neural network with one hidden layer. There are several stages to developing an ANN model. The first stage is to determine the training cycles. Training cycles are selected based on the result of the smallest RMSE. After obtaining the training cycles, the learning rate is determined to conducting a test input 
Table 2. List of input parameters.

\begin{tabular}{ccc}
\hline S/N & $\begin{array}{c}\text { Name of } \\
\text { Parameter }\end{array}$ & Description of the Parameter \\
\hline 1 & $\mathrm{~S}$ & Spot price of the security \\
2 & $\mathrm{X}$ & Exercise price of call option \\
3 & $\mathrm{R}$ & Rate of Risk-free interest \\
4 & $\mathrm{~T}$ & Time left until option expiry (date in year fraction) \\
5 & $\sigma$ & A measure of implied volatility (calculated as standard deviation) \\
\hline
\end{tabular}

value. Learning rate is also selected based on the result of the smallest RMSE. For minimizing the prediction errors, we use weight vectors randomly with input variables. In addition, we use different types of activation functions in ANN model to minimize the prediction errors. Architecture of comparing the performance of ANN and SVM models for predicting option price is illustrated in Figure 4.

\section{Experimental Results and Discussion}

For learning compositions of models ANN and SVM, we partition the information into two sections by using cross-validation, training data and testing data. Cross-validation is castoff since it ended up standard procedure in practical terms. Cross-validation makes the process to perform training 15 times because divided training data into 15 equal parts. The training and testing process in this study are performed by using MATLAB 2018a software. The parameters are optimized by the experiments through the trial and error method based on the smallest RMSE. Optimize parameters with the smallest RMSE for ANN model are reported in Table 3. It is noticed that the best results (smallest RMSE 1.743) in the experiment are found with one hidden layer, 5 inputs parameter with respective weight vectors and 4 neuron size.

The idea of SVM model can be demonstrated by considering an informational collection $\left\{\left(x_{1}, y_{1}\right),\left(x_{1}, y_{1}\right), \cdots,\left(x_{n}, y_{n}\right)\right\}$, where $x \in R^{d}$ is the $d$ dimensional input space and $y \in \mathbb{R}$ is the corresponding output. In dot function, it is defined by $k(x, y)=x * y$; where $k(x, y)$ is the inner product of $x$ and $y$. There are two parameters in the SVM model namely $C$ and Epsilon. Parameter $C$ is regularization constant, which determines the trade-off between the empirical risk and the regularization term. While the parameter Epsilon is specified as the insensitivity constant. This parameter is a part of the loss function. No loss occurs if the prediction lies this close to true value. Optimize parameters with the smallest RMSE for SVM model is summarized in Table 4. From the table, it is found that the optimum values of $\mathrm{C}$ and Epsilon are 0.1 and 0.5 , respectively. And these optimum values are found when the minimum RMSE of SVM is 1.752.

By using the optimized parameter (displayed in Table 3 and Table 4), both models are tested in the testing phase for forecasting option price in the stock 


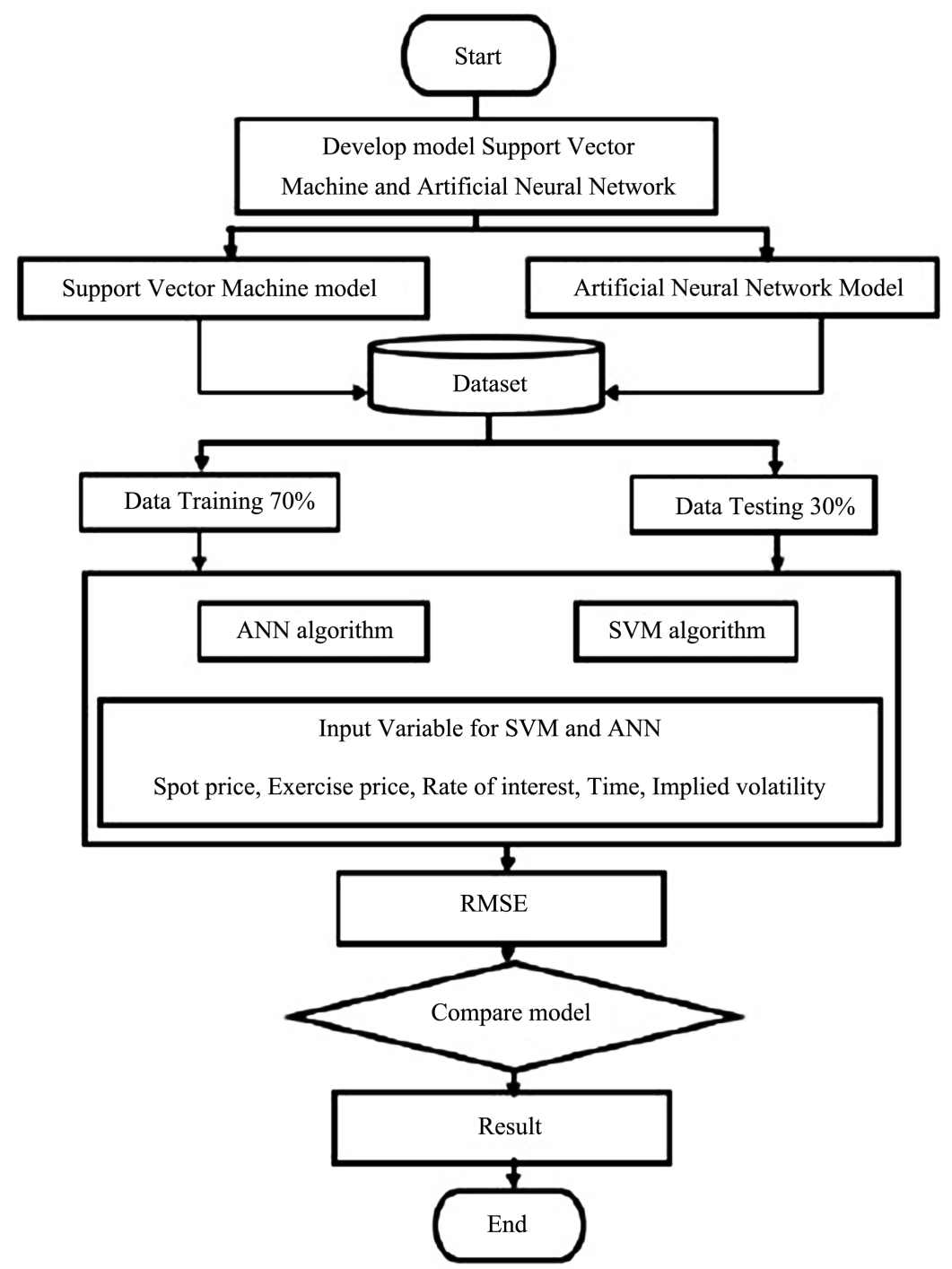

Figure 4. Architecture of comparing the performance of artificial neural network and support vector machine for predicting option price.

Table 3. Optimized parameters of artificial neural network model.

\begin{tabular}{cc}
\hline Name of Parameter & Optimize Value of Parameter \\
\hline Training Cycle & 150 \\
Learning Rate & 0.1 \\
Hidden Neuron Size & 4 \\
RMSE & 1.743 \\
\hline
\end{tabular}

Table 4. Optimized parameters of support vector machine model.

\begin{tabular}{cc}
\hline Name of Parameter & Optimize Value of Parameter \\
\hline C & 0.1 \\
Epsilon & 0.5 \\
RMSE & 1.752 \\
\hline
\end{tabular}


market. The predictive results of ANN and SVM models are displayed in Table 5. From Table 5, it can be seen that both models find the results close to the actual results. It can be also seen that the ANN model predicts the option price more accurately than the SVM model. In fact, the computed RMSE of the ANN model in this testing phase is 0.274418 , which is significantly better than the 0.409254 of the SVM model. In order to facilitate observation, the comparison of the predicted option price with the actual option price for both ANN and SVM models is illustrated graphically. This is shown in the form of scatter plot in Figure 5. From the comparison scatter plot, one can easily get an idea intuitively about the superior performance of the ANN model than the SVM model in this specific prediction problem. Therefore, it can be concluded that the ANN model might be considered as an alternative of the SVM model, which can show promising performance to predict the option price.
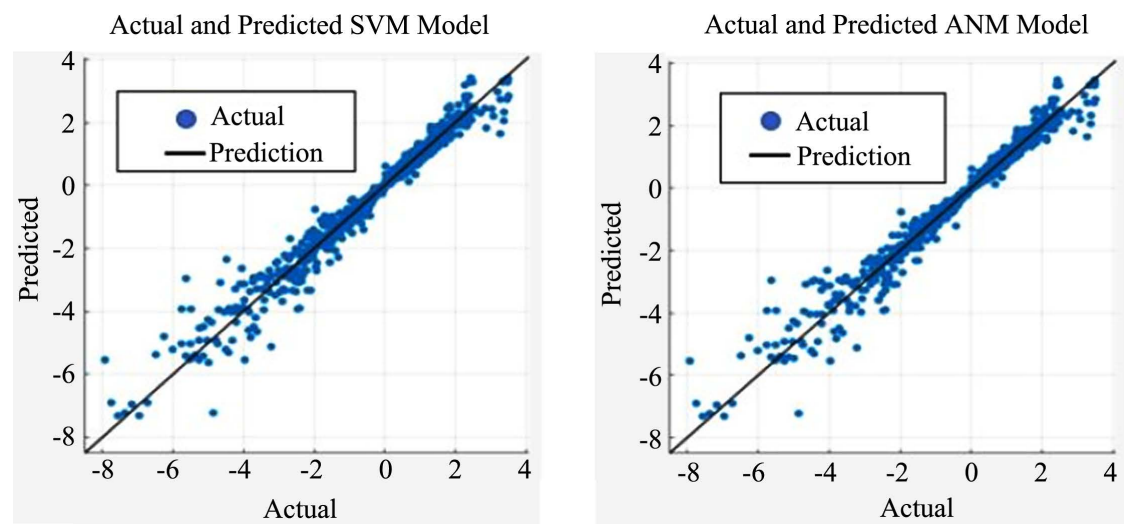

Figure 5. Scatter plot between actual and predicted price for both SVM and ANN models.

Table 5. Prediction results for option price of both ANN and SVM models.

\begin{tabular}{cccc}
\hline \multirow{2}{*}{ S/N } & Actual Price (Call/Put) & \multicolumn{2}{c}{ Predicted Price } \\
\cline { 3 - 4 } & & SVM Model & ANN Model \\
\hline 01 & 87.161 & 78.27 & 82.47 \\
02 & 82.162 & 74.47 & 79.83 \\
03 & 77.173 & 68.74 & 75.45 \\
04 & 72.171 & 65.19 & 70.92 \\
05 & 67.172 & 59.24 & 64.29 \\
06 & 62.183 & 54.77 & 59.16 \\
07 & 57.184 & 47.38 & 48.93 \\
08 & 52.192 & 39.37 & 41.43 \\
09 & 47.193 & 29.43 & 30.94 \\
. &. &. &. \\
. & $\cdot$ & $\cdot$ &. \\
4742 & $\cdot$ & 0 & 0.274418 \\
& 0 & 0.409254 &
\end{tabular}




\section{Conclusion}

Option pricing plays a very significant role in the financial market. Accurate predicting of option price helps the decision maker to take proper decision for developing better financial management. However, it still remains a challenging issue in the research community. In this paper, we have investigated the capability of two machine learning techniques such as ANN and SVM techniques to forecast option price. A decent exhibition of ANN and SVM models with performances measure indicator (RMSE) is presented in the paper. It is observed from our experiments that the ANN model yields RMSE of 1.743 and 0.274418 in training and testing stages respectively, which are better than the 1.752 and 0.409254 of SVM model. The experimental results indicate that the ANN model outperforms SVM model for predicting option price. The experimental results also suggest that the ANN model is a promising technique and it can be adopted as an alternative of the SVM model in predicting option price at this particular area. However, further research needs to be accomplished to identify the strength and weakness of this model.

\section{Conflicts of Interest}

The authors declare no conflicts of interest regarding the publication of this paper.

\section{References}

[1] Hoti, S., McAleer, M. and Pauwels, L.L. (2008) Multivariate Volatility in Environmental Finance. Mathematics and Computers in Simulation, 78, 189-199. https://doi.org/10.1016/j.matcom.2008.01.038

[2] Wen, Q., Yang, Z., Song, Y. and Jia, P. (2010) Automatic Stock Decision Support System Based on Box Theory and SVM Algorithm. Expert Systems with Applications, 37, 1015-1022. https://doi.org/10.1016/j.eswa.2009.05.093

[3] Kampouridis, M., Alsheddy, A. and Tsang, E. (2013) On the Investigation of Hyper-Heuristics on a Financial Forecasting Problem. Annals of Mathematics and Artificial Intelligence, 68, 225-246. https://doi.org/10.1007/s10472-012-9283-0

[4] Tan, T. Z., Quek, C. and Ng, G. S. (2005) Brain-Inspired Genetic Complementary Learning for Stock Market Prediction. 2005 IEEE Congress on Evolutionary Computation, Vol. 3, Edinburgh, 2-5 September 2005, 2653-2660. https://doi.org/10.1109/CEC.2005.1555027

[5] Yu, L., Wang, S. and Lai, K.K. (2009) A Neural-Network-Based Nonlinear Metamodeling Approach to Financial Time Series Forecasting. Applied Soft Computing, 9, 563-574. https://doi.org/10.1016/j.asoc.2008.08.001

[6] Liu, M. (1996) Option Pricing with Neural Networks. In: Amari, S.I., Xu, L., Chan, L.W., King, I. and Leung, K.S., Eds., Progress in Neural Information Processing, Vol. 2, Springer-Verlag, New York, 760-765.

[7] Yao, J., Li, Y. and Tan, C.L. (2000) Option Price Forecasting Using Neural Networks. Omega, 28, 455-466. https://doi.org/10.1016/S0305-0483(99)00066-3

[8] Andreou, P.C., Charalambous, C. and Martzoukos, S.H. (2008) Pricing and Trading European Options by Combining Artificial Neural Networks and Parametric Mod- 
els with Implied Parameters. European Journal of Operational Research, 185, 14151433. https://doi.org/10.1016/j.ejor.2005.03.081

[9] Zhang, Y. and Wu, L. (2009) Stock Market Prediction of S\&P 500 via Combination of Improved BCO Approach and BP Neural Network. Expert Systems with Applications, 36, 8849-8854. https://doi.org/10.1016/j.eswa.2008.11.028

[10] Olatunji, S.O., Al-Ahmadi, M.S., Elshafei, M. and Fallatah, Y.A. (2011) Saudi Arabia Stock Prices Forecasting Using Artificial Neural Networks. 4th International Conference on the Applications of Digital Information and Web Technologies (ICADIWT 2011), Stevens Point, 4-6 August 2011, 81-86. https://doi.org/10.1109/ICADIWT.2011.6041425

[11] Chang, T.S. (2011) A Comparative Study of Artificial Neural Networks, and Decision Trees for Digital Game Content Stocks Price Prediction. Expert Systems with Applications, 38, 14846-14851. https://doi.org/10.1016/j.eswa.2011.05.063

[12] Saxena, A. (2008) Valuation of S\&P CNX Nifty Options: Comparison of Black-Scholes and Hybrid ANN Model. Proceedings of SAS Global Forum, San Antonio, 16-19 March 2008, 1-25.

[13] Mitra, S.K. (2012) An Option Pricing Model That Combines Neural Network Approach and Black Scholes formula. Global Journal of Computer Science and Technology, 12, 1-11.

[14] Lajbcygier, P.R. and Connor, J.T. (1997) Improved Option Pricing Using Artificial Neural Networks and Bootstrap Methods. International Journal of Neural Systems, 8, 457-471. https://doi.org/10.1142/S0129065797000446

[15] Hassan, M.R., Nath, B. and Kirley, M. (2007) A Fusion Model of HMM, ANN and GA for Stock Market Forecasting. Expert Systems with Applications, 33, 171-180. https://doi.org/10.1016/j.eswa.2006.04.007

[16] Kara, Y., Boyacioglu, M.A. and Baykan, Ö.K. (2011) Predicting Direction of Stock Price Index Movement Using Artificial Neural Networks and Support Vector Machines: The Sample of the Istanbul Stock Exchange. Expert Systems with Applications, 38, 5311-5319. https://doi.org/10.1016/j.eswa.2010.10.027

[17] Zhu, X., Wang, H., Xu, L. and Li, H. (2008) Predicting Stock Index Increments by Neural Networks: The Role of Trading Volume under Different Horizons. Expert Systems with Applications, 34, 3043-3054. https://doi.org/10.1016/j.eswa.2007.06.023

[18] Chen, W.H., Shih, J.Y. and Wu, S. (2006) Comparison of Support-Vector Machines and Back Propagation Neural Networks in Forecasting the Six Major Asian Stock Markets. International Journal of Electronic Finance, 1, 49-67. https://doi.org/10.1504/IJEF.2006.008837

[19] Li, P., Tan, Z., Yan, L. and Deng, K. (2011) Time Series Prediction of Mining Subsidence Based on a SVM. Mining Science and Technology (China), 21, 557-562. https://doi.org/10.1016/j.mstc.2011.02.025

[20] Hutchinson, J.M., Lo, A.W. and Poggio, T. (1994) A Nonparametric Approach to Pricing and Hedging Derivative Securities via Learning Networks. The Journal of Finance, 49, 851-889. https://doi.org/10.1111/j.1540-6261.1994.tb00081.x

[21] Kim, K.J. (2003) Financial Time Series Forecasting Using Support Vector Machines. Neurocomputing, 55, 307-319. https://doi.org/10.1016/S0925-2312(03)00372-2

[22] Yoshihara, A., Fujikawa, K., Seki, K. and Uehara, K. (2014) PREDICTING stock market Trends by Recurrent Deep Neural Networks. Pacific Rim International Conference on Artificial Intelligence, Gold Coast, 1-5 December 2014, 759-769. 
https://doi.org/10.1007/978-3-319-13560-1_60

[23] Lekhani, R. (2017) Stock Prediction Using Support Vector Regression and Neural Networks. International Journal of Advance Research, Ideas and Innovations in Technology, 3, 441-445.

[24] Madhu, B., Paul, A.K. and Roy, R. (2019) Performance Comparison of Various Kernels of Support Vector Regression for Predicting Option Price. International Journal of Discrete Mathematics, 4, 21-31. https://doi.org/10.11648/j.dmath.20190401.14

[25] https://dlpng.com/png/6583820

[26] https://finance.yahoo.com/quote/spy/options 